\title{
Molecular characterization of Cryptosporidium isolates from humans in Ontario, Canada
}

Rebecca A. Guy ${ }^{1 *} \mathbb{D}$, Christine A. Yanta ${ }^{1}$, Pia K. Muchaal ${ }^{2}$, Marisa A. Rankin ${ }^{1}$, Karine Thivierge ${ }^{3}$, Rachel Lau ${ }^{4}$ and Andrea K. Boggild $4,5,6$

\begin{abstract}
Background: Cryptosporidiosis is a gastrointestinal disease with global distribution. It has been a reportable disease in Canada since 2000; however, routine molecular surveillance is not conducted. Therefore, sources of contamination are unknown. The aim of this project was to identify species and subtypes of Cryptosporidium in clinical cases from Ontario, the largest province in Canada, representing one third of the Canadian population, in order to understand transmission patterns.
\end{abstract}

Methods: A total of 169 frozen, banked, unpreserved stool specimens that were microscopy positive for Cryptosporidium over the period 2008-2017 were characterized using molecular tools. A subset of the 169 specimens were replicate samples from individual cases. DNA was extracted directly from the stool and nested PCR followed by Sanger sequencing was conducted targeting the small subunit ribosomal RNA (SSU) and glycoprotein 60 (gp60) genes.

Results: Molecular typing data and limited demographic data were obtained for 129 cases of cryptosporidiosis. Of these cases, 91 (70.5 \%) were due to Cryptosporidium parvum and 24 (18.6\%) were due to Cryptosporidium hominis. Mixed infections of $C$. parvum and C. hominis occurred in four (3.1\%) cases. Five other species observed were Cryptosporidium ubiquitum $(n=5)$, Cryptosporidium felis $(n=2)$, Cryptosporidium meleagridis $(n=1)$, Cryptosporidium cuniculus $(n=1)$ and Cryptosporidium muris $(n=1)$. Subtyping the gp60 gene revealed 5 allelic families and 17 subtypes of C. hominis and 3 allelic families and 17 subtypes of C. parvum. The most frequent subtype of C. hominis was IbA10G2 (22.3\%) and of C. parvum was IlaA15G2R1 (62.4\%).

Conclusions: The majority of isolates in this study were C. parvum, supporting the notion that zoonotic transmission is the main route of cryptosporidiosis transmission in Ontario. Nonetheless, the observation of $C$. hominis in about a quarter of cases suggests that anthroponotic transmission is also an important contributor to cryptosporidiosis pathogenesis in Ontario.

Keywords: Coccidiosis, Cryptosporidium parvum, Cryptosporidium hominis, Gp60, Sanger sequencing, Zoonosis

\footnotetext{
*Correspondence: rebecca.guy@canada.ca

${ }^{1}$ Parasite Biology Unit/Division of Enteric Diseases, National Microbiology

Laboratory, Public Health Agency of Canada, 110 Stone Road West, Guelph, ON N1G 3W4, Canada

Full list of author information is available at the end of the article
}

\section{Background}

Cryptosporidium is a protozoan parasite that causes gastrointestinal disease worldwide and is a major cause of severe morbidity and mortality in young children in developing countries [1]. In 2016, it was the leading cause of diarrheal mortality in children under 5 years old with 48,000 deaths due to acute infection [2]. Cryptosporidium can be transmitted indirectly through ingestion of

(C) The Author(s) 2021. This article is licensed under a Creative Commons Attribution 4.0 International License, which permits use, sharing, adaptation, distribution and reproduction in any medium or format, as long as you give appropriate credit to the original author(s) and the source, provide a link to the Creative Commons licence, and indicate if changes were made. The images or other third party material in this article are included in the article's Creative Commons licence, unless indicated otherwise in a credit line to the material. If material is not included in the article's Creative Commons licence and your intended use is not permitted by statutory regulation or exceeds the permitted use, you will need to obtain permission directly from the copyright holder. To view a copy of this licence, visit http://creativeco mmons.org/licenses/by/4.0/. The Creative Commons Public Domain Dedication waiver (http://creativecommons.org/publicdomain/ zero/1.0/) applies to the data made available in this article, unless otherwise stated in a credit line to the data. 
contaminated food or water, or directly from person to person or animal to person. Infective oocysts are stable in the environment for long periods, are resistant to standard disinfection strategies such as chlorination and iodination, and thus are easily transmitted in water.

Cryptosporidiosis has been a reportable disease in Canada since 2000. Since then, reported annual laboratory-confirmed cases [3] number only 723 on average, but due to the constellation of under-diagnostics and significant under-reporting, estimated annual cases are 35,092 [4]. Higher rates of cryptosporidiosis have been reported in the arctic region of Canada [5-7]. National reporting in the UK, Ireland, France, Sweden and the USA has revealed national and regional differences in species distribution and subtypes of Cryptosporidium in humans and high under-ascertainment of this disease [8].

In Canada, as in most countries, national reporting requirements are to the genus level (Cryptosporidium spp.); hence, information regarding transmission of sporadic cases through zoonotic or anthroponotic routes is unknown. There are over 38 species of Cryptosporidium, 2 of which are major species identified in humans, Cryptosporidium hominis and Cryptosporidium parvum, and 21 other species of Cryptosporidium infect humans less frequently [9]. In developing countries, the human host-adapted $C$. hominis predominates, whereas both $C$. parvum and $C$. hominis are observed in developed countries [8], with a predominance of C. parvum in parts of Europe [10-13], Northern USA [14-16] and Canada [7, 17].

Understanding the species and subtypes of disease causing Cryptosporidium requires molecular analysis of the parasite. A handful of studies have used molecular typing to identify Cryptosporidium species and genotypes in humans in Canada, for source attribution and mitigation of infection. Similar zoonotic C. parvum gp60 subtypes among humans and cattle were reported in southwestern Ontario [18] and in Prince Edward Island (PEI) [17]. These studies were limited because of the very low number of samples suitable for PCR analysis because in Canada stool is routinely placed into formalin-based fixatives for microscopy analysis of parasites. Some first-line laboratories are moving to molecular diagnosis methods, which may lead to enhanced genotyping when parasite testing is requested. Recently, high rates of $C$. parvum were reported in humans in Quebec [7] with similar zoonotic subtypes as reported in Ontario and PEI $[17,18]$.

Analysis of 36 endemic cryptosporidiosis cases from 2005-2007 in the Ontario FoodNet Canada sentinel site identified several risk factors for cryptosporidiosis: swimming in a lake or river, consuming municipal drinking water and/or having a family member with a diarrheal illness [19]. Passive surveillance data from 161 Ontario cases with exposure data between 2007 and 2009 determined primary exposures for infection to be associated with animal (47.2\%), food (9.3\%), person-to-person $(11.8 \%)$ or water $(29.2 \%)$ transmission [20]. No laboratory confirmation of species and subtype were available to support source and transmission routes for those studies.

The aim of this study is to better understand reservoirs of infection and transmission dynamics in Ontario. Ontario is the most populous jurisdiction of the ten provinces and three territories in Canada, with a reported 14.7 million people in 2019 [https://doi.org/10.25318 /1710000901-eng], comprising $38.8 \%$ of Canadians. It is home to the largest urban centers in the country, with cities such as Toronto and Mississauga, as well as large regions of agriculture/livestock production, especially in the southwest of the province. Thus, potential for exposure to diverse Cryptosporidium species and subtypes exists in Ontario. Annual reported cases of cryptosporidiosis in Ontario, between 2008 and 2017, ranged from 299 to 469 cases [21] $]^{1}$.

The objectives of this study were to identify the species and subtypes of Cryptosporidium in sporadic cases from a random subset of Cryptosporidium-positive human stool samples from 2008-2017, submitted to the provincial reference laboratory in specimen collection containers lacking fixative. Through molecular analysis of the small subunit ribosomal RNA (SSU) and gp60 gene loci, our aim was to gain a better understanding of potential sources of infection of Cryptosporidium in Ontarians.

\section{Methods \\ Samples}

Samples used for molecular analysis in this study were surplus unpreserved stool specimens positive for Cryptosporidium spp. by routine microscopic fecal examination (including light microscopic examination of iron-haematoxylin stained smears and formalin-ethyl acetate concentrates, as well as auramine-rhodamine fluorescence microscopy) during the period of 2008-2017. These samples were identified and retrieved from the $-20{ }^{\circ} \mathrm{C}$ biobank at the Public Health Ontario Laboratory. Demographic information, including age, sex, travel history and clinical symptoms, were obtained, provided by the ordering physician on the standard Public Health Ontario Laboratory test requisition (https://www.publichealthont ario.ca/-/media/documents/lab/general-test-requisitio n.pdf?la $=$ en).

\footnotetext{
${ }^{1}$ Cases reported in Ontario include laboratory-confirmed cases and epidemiological cases linked to a laboratory-confirmed case.
} 


\section{Ethics}

Ethics approval was granted by the Research Ethics Board of Health Canada/Public Health Agency of Canada (Ethics certificate REB 2016-010P). Specimens were transferred to the Public Health Agency of Canada from Public Health Ontario in accordance with a Material Transfer Agreement (MTA 16-033).

\section{DNA extraction}

Frozen fecal samples were stored at $-20{ }^{\circ} \mathrm{C}$. Following thawing, the stool was mixed, and a $0.2 \mathrm{~g}$ aliquot of stool was processed using the QIAamp Fast DNA Mini Stool kit (Qiagen, Hilden, Germany). The manufacturers' instructions were followed with this exception: after incubation in InhibitEx buffer plus proteinase $\mathrm{K}$ at $56{ }^{\circ} \mathrm{C}$ for $1 \mathrm{~h}$, the samples were subjected to five consecutive cycles of freeze/thaw for 2 min each in liquid nitrogen and in a dry bath at $65{ }^{\circ} \mathrm{C}$. DNA was eluted in two rounds of $50 \mu \mathrm{l} \mathrm{AE}$ buffer and stored at $-20^{\circ} \mathrm{C}$ until use.

\section{Nested PCR (nPCR)}

All samples were analyzed at the SSU and gp60 loci using nested PCR. Platinum Taq (Invitrogen by Life Technologies, Carlsbad, CA, USA) was used as the polymerase and bovine serum albumin, heat shock fraction (SigmaAldrich, St Louis, MO, USA) was added at a final concentration of $300 \mathrm{ng} / \mu \mathrm{l}$ to all primary reactions to reduce PCR inhibition. For the SSU assays, primer concentrations used were as previously described [22]. PCR reactions were carried out in $50 \mu \mathrm{l}$ reactions, and $2 \mu \mathrm{l}$ of the primary product was used in the secondary PCR. Samples that were negative in repeated SSU nPCR were analyzed using an HSP70 nPCR assay [23]. Part way through the study, screening for C. parvum (LIB13 locus) and $C$. hominis (A135 locus) was performed using a multiplex real-time PCR assay [24].

The gp60 PCR was conducted in $100 \mu \mathrm{l}$ reactions using species-specific primers for C. parvum, C. hominis and Cryptosporidium cuniculus [25], Cryptosporidium felis [26], Cryptosporidium meleagridis [27] and Cryptosporidium ubiquitum [28]. Amplification was conducted on a Biometra instrument using the cycling conditions in the original articles. Samples were run in duplicate, and nPCR was repeated if the first nPCR was negative. PCR fragments were analyzed using capillary electrophoresis on a QIAxcel DNA Screening cartridge (Qiagen, Hilden, Germany) using the AM420 method. Qiagen's QX 15bp3kb Alignment Marker and 50-800 bp size marker were run with the samples.

\section{Sequencing}

Positive samples were purified using the MinElute purification kit (Qiagen, Hilden, Germany) prior to bidirectional Sanger sequencing performed at the CRCHUL sequencing center in Quebec City. Sequence chromatograms were analyzed using BioEdit and MEGA6 programs and on our newly developed SSU and gp60 typer program called CryptoGenotyper [29, submitted]. Fasta files for each sequence were analyzed using BLAST (Basic Local Alignment Search Tool, NCBI) analysis. Naming of subtypes was based on the scheme described by Xiao [30].

\section{Nucleotide sequence accession numbers}

Unique and rare, partial gp60 sequences were deposited in GenBank under accession numbers MT953499-MT953508.

\section{Data analysis}

Data including age, sex, travel history and month of primary routine clinical testing were obtained and housed in an encrypted, password-protected Excel file to which only the investigators had access. Analyses were performed to describe the age, sex and seasonal distribution of Cryptosporidium species and subtypes. Differences in cases by causative agent (i.e. species of Cryptosporidium) were determined using the non-parametric KruskalWallis test. The Fischer's exact test for significance was applied for the analysis of categorical variables where cell counts were $<5$. All statistical analyses were performed using SAS version 9.4. Level of significance was set at $p$ $<0.05$.

\section{Results \\ Identification of Cryptosporidium in clinical specimens}

In Ontario, stool specimens for ova and parasite examination are routinely submitted in a fixative solution of sodium acetate, acetic acid and formalin (SAF). Unfixed stool specimens were used in this study and represented only $9.0 \%(169 / 1868)$ of the total number of specimens submitted during the period 2010-2017. From 2010-2017, there were $0 \%(0 / 172), 3.3 \%(7 / 211), 14.9 \%$ (30/202), 7.1\% (14/198), 5.0\% (13/258), 17.4\% (47/270), $9.8 \%(30 / 305)$ and $10.3 \%(26 / 252)$ unpreserved specimens per year, respectively. This study included two specimens from 2008 and one from 2009; however, the total number of specimens for those years was unavailable.

The 169 Cryptosporidium-positive human clinical stool specimens analyzed represented 131 cases of cryptosporidiosis diagnosed between 2008 and 2017. Duplicate and triplicate samples were present for 14 cases. Testing of replicates for ten of the cases resulted in the same species and subtypes identified in all replicates per case, for both the SSU and gp60 genes. The success rates for SSU and gp60 typing were $99.2 \%(130 / 131)$ and $95.4 \%$ (125/131), respectively. The one case not identified using 
the SSU nPCR was identified as C. felis using an HSP70 nPCR.

Of the 131 cases, 129 individual cases had associated demographic and molecular data. There were a variable number of cases for each year: $2008(n=2), 2009(n=1)$, $2011(n=5), 2012(n=24), 2013(n=10), 2014(n=5)$, $2015(n=35), 2016(n=29)$ and $2017(n=19)$. Among the 129 cases, seven species of Cryptosporidium were identified, with $C$. parvum and $C$. hominis representing 92.2\% (119/129) of the cases (Table 1). There were four mixed infections of $C$. parvum and $C$. hominis and five other species that occurred at low frequencies (Table 1). A total of $50.4 \%(n=65)$ of cases were female and $49.6 \%$ ( $n=64$ ) were male. Among C. hominis infections, there was a significant difference $\left(\chi^{2}=4.532, \mathrm{df}=1, P=\right.$ $0.0333)$ in the age distribution of female cases with $C$. hominis infections (median age $=31$ years, range $=7-78$ years) compared to males (median age $=9$ years, range $=1-62)$. By contrast, no difference $\left(\chi^{2}=2.885, \mathrm{df}=1\right.$, $P=0.0844$ ) was noted between infections of $C$. parvum in females (median age $=24$ years, range $2-87$ years) and males (median age $=19.5$ years, range $=2-59$ years).

\section{Age distribution of Cryptosporidium in males and females}

For all cases of $C$. parvum and C. hominis, the median age was 23 years (range $=1-87$ years), and the proportion of female and male cases varied by age group (Fig. 1). The median age of female cases was 25 years (range $=$ 2-87 years) compared to 18 years for males (range $=$ 1-62 years). Among children/youth $<20$ years of age, there were fewer cases of girls compared to boys, a ratio of 0.58 . By contrast, there were 1.6 times as many females compared to males in those aged 20-35 years. The median age of the five C. ubiquitum cases was 4 (range $=$ $1-18)$ years.

\section{Reported symptoms}

Symptom information was available for $62 \%$ of cases (82/129). Diarrhea was identified as the only symptom in $36.6 \%$ of cases $(30 / 82)$ and gastroenteritis in $53.7 \%$ of cases (44/82). For the purposes of this analysis, it was assumed that gastroenteritis refers to diarrhea or vomiting. In $67.2 \%$ of cases (39/58) infected with C. parvum subtype IIaA15G2R1, the cases experienced gastroenteritis and/or diarrhea or vomiting.

\section{Identification of gp60 subtypes}

Subtyping the gp60 gene revealed a large genetic diversity of Cryptosporidium infections among cases of $C$. hominis (5 allelic families, 17 subtypes) and C. parvum (3 allelic families, 17 subtypes) (Table 2). Only a single
Table 1 Percent SSU PCR positive species identification of Cryptosporidium in 129 female and male cryptosporidiosis cases in Ontario from 2008-2017

\begin{tabular}{|c|c|c|c|c|}
\hline \multirow[t]{2}{*}{ Cryptosporidium species } & \multicolumn{2}{|c|}{$\begin{array}{l}\text { Total } \\
\text { cases }\end{array}$} & \multirow{2}{*}{$\begin{array}{l}\text { Females }(n=65) \\
\%\end{array}$} & \multirow{2}{*}{$\begin{array}{l}\text { Males }(n=64) \\
\%\end{array}$} \\
\hline & $n$ & $\%$ & & \\
\hline C.parvum & 91 & 70.5 & 36.4 & 34.1 \\
\hline C. hominis & 24 & 18.6 & 9.3 & 9.3 \\
\hline C. ubiquitum & 5 & 3.9 & 1.6 & 2.3 \\
\hline C. felis & 2 & 1.6 & 0.0 & 1.6 \\
\hline C. meleagridis & 1 & 0.8 & 0.8 & 0.0 \\
\hline C. cuniculus & 1 & 0.8 & 0.0 & 0.8 \\
\hline C.muris & 1 & 0.8 & 0.8 & 0.0 \\
\hline C.parvum/C. hominis & 4 & 3.1 & 1.6 & 1.6 \\
\hline
\end{tabular}

Not included in Table: one case of C. parvum and one of C. hominis of unknown gender

gp60 subtype was observed in three of the four mixed C. parvum/C. hominis cases: IbA12G3, IIcA5G3a and IIaA15G2R1. One mixed C. parvum/C. hominis case had both IfA12G1R4 and IIcA5G3a gp60 subtypes. One $C$. parvum case had mixed IIaA15G2R1 and IIaA16G1R1 subtypes. Two cases of C. ubiquitum were subtype XIIb, two cases were XIId, and one case was untypable. One of the two C. felis isolates was typed as subtype XIXd-1. The C. cuniculus case was subtype VbA38, and the C. meleagridis case was IIIeA21G2.

\section{gp60 subtypes in travel cases}

Only a handful of cases, $3.1 \%$ (4/129), were reportedly acquired during travel with known locations for three (75\%) of the travel cases. These corresponded to C. parvum IlaA19G1R1 acquired on travel to Romania, C. parvum IIaA17G2R1 acquired in Taiwan and C. hominis IbA10G2 acquired in Lebanon, and the single $C$. meleagridis case was acquired during travel to an undisclosed location.

\section{Seasonal distribution of $g p 60$ subtypes}

The proportion of samples that we obtained for genotyping from cases in the 9-year study period ranged from 0.01 to $0.1(1-10 \%)$ of the total cases per month reported in Ontario during that period [21]. Cryptosporidiosis cases peaked in the summer, with most of the cases occurring in August (Fig. 2a). A similar trend was observed in individual years (not shown). A different trend was seen when looking at the seasonal distribution of cases with specimens that were subtyped (Fig 2a), with peaks in the winter and fall.

Plotting the subset of samples that were genotyped at the gp60 locus revealed that C. parvum cases were 


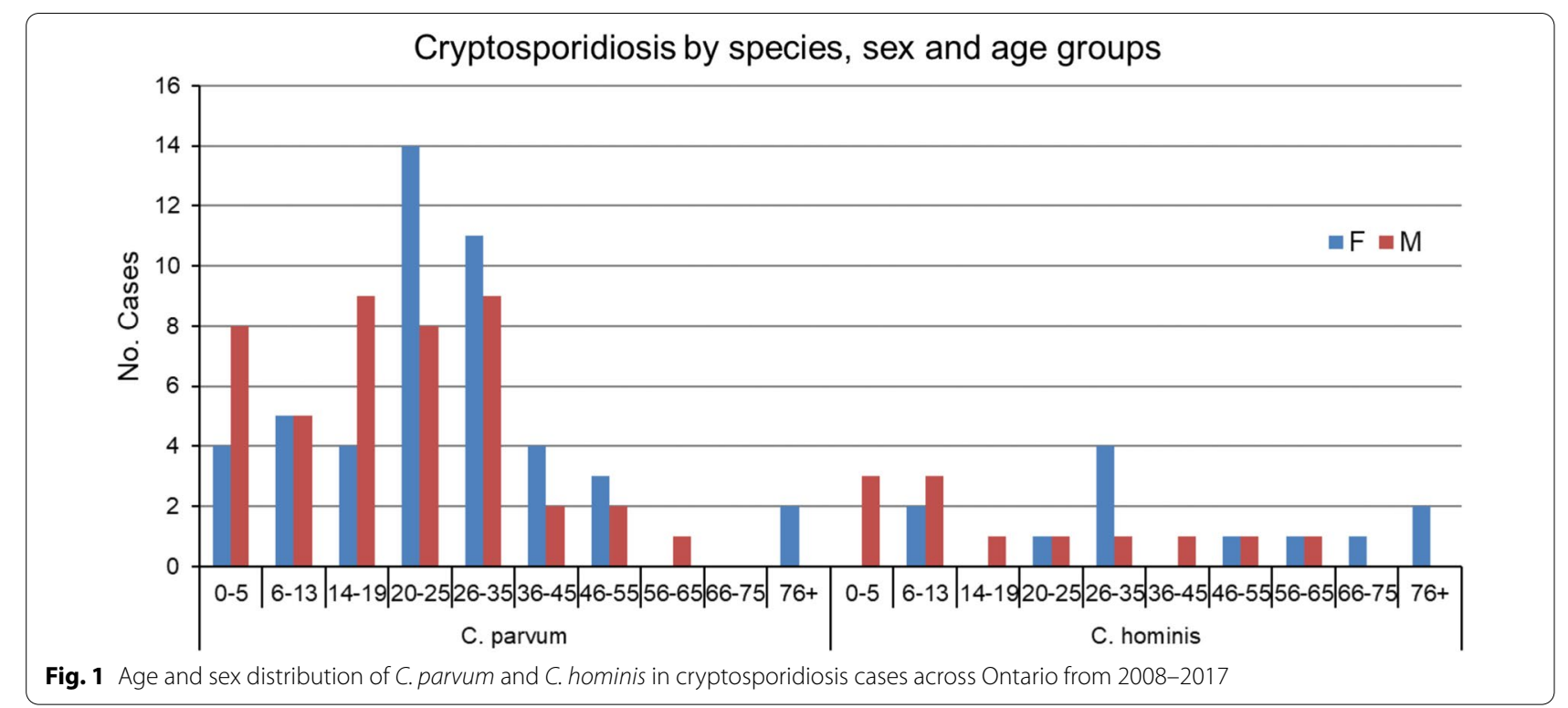

detected throughout the year with a summer peak and a moderate increase during late winter and early spring (Fig. 2b). Cryptosporidium parvum IIaA15G2R1 was the most common subtype identified, comprising $62.4 \%$ of all C. parvum cases, and was the predominant subtype of C. parvum and C. hominis combined in 6 of the 9 study years (Table 3 ).

Cases of the IIaA15G2R1 subtype occurred throughout the year, with the exception of April and September, and constituted $12.5 \%$ to $87.5 \%$ of cryptosporidiosis cases due to $C$. parvum and $C$. hominis diagnosed each month (Fig. 2b). IIaA17G2R1, the second most frequently observed subtype of C. parvum, was only noted in cases that occurred in the second half of the year. Cryptosporidium hominis subtypes were observed from February through to October and in December (Fig. 2c). IbA10G2, the most commonly diagnosed subtype of $C$. hominis, occurred in August, September and December. The peak in cases during the summer period was also observed in individual years, 2012 and 2015 (Fig. 3a, b). Higher winter cases were observed in 2016 and 2017 (Fig. 3c, d).

\section{gp60 subtypes reported in Canada}

A summary of published gp60 subtypes in humans in Canada (Table 4) shows that of the 204 isolates with gp60 subtypes reported in the literature, there were 26 subtypes of $C$. hominis and 19 subtypes of C. parvum. Twenty-two new subtypes $(12 \mathrm{C}$. hominis and 10 C. parvum) were identified in the present study that were not previously reported in Canada (Table 4). Subtype IIaA15G2R1 (36.8\%) was the most common subtype, followed by IbA10G2 (6.9\%) IIaA17G2R1 (5.9\%), IIaA16G3R1 (5.4\%) and IdA19 (5.4\%). Subtyping in two of these studies was performed on outbreak specimens $[6,31]$.

\section{Discussion}

This study identified species and subtypes of Cryptosporidium on a larger scale than previously reported from Canadians. Based on the total number of samples analyzed at Public Health Ontario each year, the samples tested herein represent a subset ranging from $3.3-17.4 \%$ of the submitted specimens per year from 2008-2017. Despite the small sample size, the typing results reveal a diversity of Cryptosporidium subtypes detected in Ontario and thus variable potential sources of contamination and transmission routes. Longitudinal molecular surveillance of isolates for species and subtype identification of cryptosporidiosis cases in Europe, Australia and North America [11, 25, 32, 33] highlights the role of molecular testing in surveillance and outbreak detection for prevention and mitigation of cryptosporidiosis.

The distribution of species detected in the Ontario cryptosporidiosis cases mirrors a study in PEI, the province with the highest cattle-to-human ratio in Canada, where $64 \%$ of the isolates were C. parvum and $37 \%$ were C. hominis [17], similar to levels reported in Scotland and Sweden $[11,12]$. Equal numbers of $C$. parvum and $C$. hominis were observed in an earlier Ontario study [18]. Higher percentages of C. parvum (74\%) than C. hominis (23\%) were recently reported from a 2016-2017 study of cryptosporidiosis in Quebec, a province of 8.5 million people that neighbors Ontario. Very high percentages 
Table 2 Cryptosporidium hominis and Cryptosporidium parvum gp60 subtypes detected in cryptosporidiosis cases in Ontario from 2008-2017

\begin{tabular}{|c|c|c|c|c|c|c|}
\hline \multirow[t]{2}{*}{ Species (no. subtypes) } & \multirow[t]{2}{*}{ Subtype family } & \multicolumn{2}{|c|}{ Total subtype family } & \multicolumn{3}{|c|}{ Total subtypes } \\
\hline & & $n$ & $\%$ & Subtype & $n$ & $\%$ \\
\hline \multirow[t]{17}{*}{ C. hominis $(n=27)$} & la & 4 & 14.8 & laA14R3 & 1 & 3.7 \\
\hline & & & & laA19R3 & 1 & 3.7 \\
\hline & & & & laA24R4 & 1 & 3.7 \\
\hline & & & & laA25R3 & 1 & 3.7 \\
\hline & lb & 9 & 33.3 & IbA9G3 & 2 & 7.4 \\
\hline & & & & $\mathrm{IbA} 10 \mathrm{G} 2$ & 6 & 22.3 \\
\hline & & & & $\mathrm{IbA} 12 \mathrm{G} 3$ & 1 & 3.7 \\
\hline & ld & 7 & 25.9 & $\mid \mathrm{dA} 14$ & 1 & 3.7 \\
\hline & & & & $\mathrm{IdA} 15$ & 2 & 7.4 \\
\hline & & & & IdA15G1 & 1 & 3.7 \\
\hline & & & & $\mid d A 17$ & 1 & 3.7 \\
\hline & & & & IdA22 & 1 & 3.7 \\
\hline & & & & $\mathrm{IdA} 26$ & 1 & 3.7 \\
\hline & le & 2 & 7.4 & leA11G3T3 & 2 & 7.4 \\
\hline & If & 5 & 18.5 & IfA12G1R4 & 1 & 3.7 \\
\hline & & & & IfA12G1R5 & 3 & 11.1 \\
\hline & & & & IfA14G1R5 & 1 & 3.7 \\
\hline \multirow[t]{17}{*}{ C. parvum $(n=94)$} & Ila & 86 & 91.4 & IlaA13G2R1 & 1 & 1.1 \\
\hline & & & & IlaA14G1R1 & 1 & 1.1 \\
\hline & & & & IlaA14G2R1 & 1 & 1.1 \\
\hline & & & & IlaA15G2R1 & 58 & 61.6 \\
\hline & & & & IlaA15G2R2 & 3 & 3.2 \\
\hline & & & & IlaA16G1R1 & 1 & 1.1 \\
\hline & & & & IlaA16G2R1 & 2 & 2.1 \\
\hline & & & & IlaA16G3R1 & 6 & 6.3 \\
\hline & & & & IlaA17G2R1 & 8 & 8.4 \\
\hline & & & & IlaA17G3R1 & 1 & 1.1 \\
\hline & & & & IlaA17G4R1 & 2 & 2.1 \\
\hline & & & & IlaA19G1R1 & 1 & 1.1 \\
\hline & & & & IlaA19G2R1 & 1 & 1.1 \\
\hline & IIc & 4 & 4.3 & IlcA5G3a & 3 & 3.2 \\
\hline & & & & $11 \mathrm{cA} 5 \mathrm{G} 3 \mathrm{~d}$ & 1 & 1.1 \\
\hline & Ild & 4 & 4.3 & ॥dA18G1 & 1 & 1.1 \\
\hline & & & & ॥dA20G1 & 3 & 3.2 \\
\hline
\end{tabular}

Two cases with subtype laA25R3 and IlaA15G2R1, excluded in Table 1 because of a lack of demographic data, are included in this Table. Two cases of C. parvum were not typable; one case of mixed C. hominis/C. parvum had mixed gp60 subtypes IfA12G1R4 and IlcA5G3a; one case of C. parvum contained mixed gp60 subtypes of IlaA15G2R1 and IlaA16G1

of C. parvum were previously reported in Ireland, Denmark and the Northwest USA [10, 13, 15]. Conversely, higher percentages of $C$. hominis occur in much of the USA, Australia and Spain [25, 33, 34]. Longitudinal studies have shown changes in the ratios of C. parvum/C. hominis over time in England and Wales, France and The Netherlands [35-38], highlighting the importance of longitudinal surveillance.
Differences in the age distribution of Cryptosporidium cases are well documented with a peak in children $<5$ years of age [10,33,39] or $<9$ years of age [11, 32, 35]. This was also observed in this study, in other studies in Ontario [19, 20] and Quebec [7] and in outbreaks in Northern Canada [6]. A peak at 5-10 years of age was reported in PEI [17]. A second peak, in adults 30-39 years old, is also widely reported [11, 38, 39]. We also 


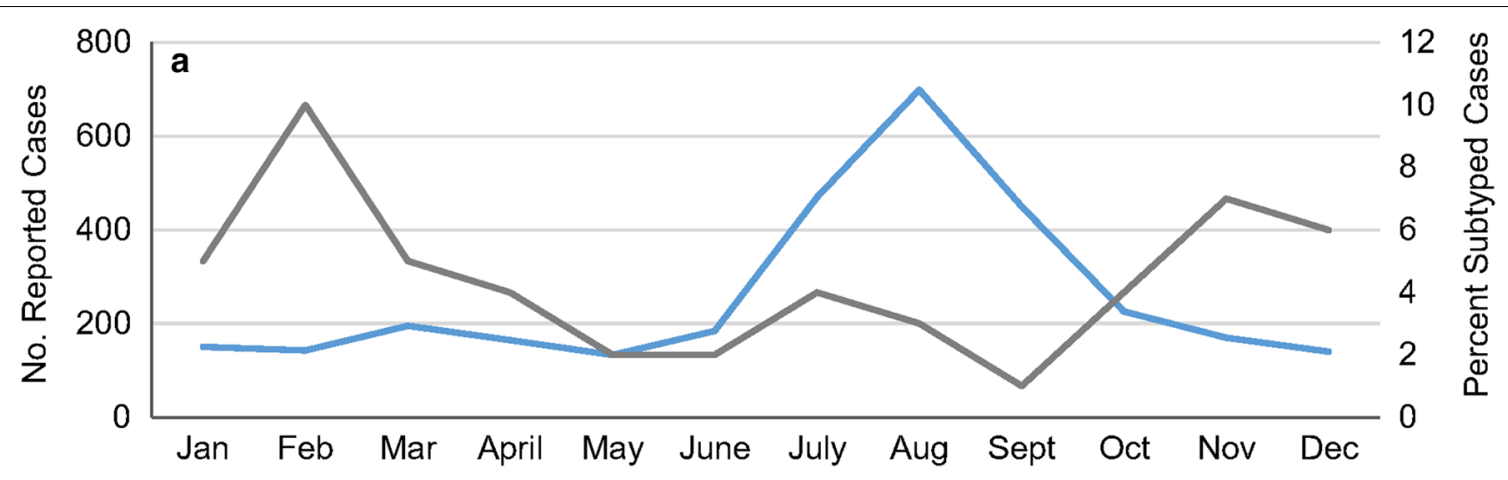

Total Cases $\quad$ Subtyped Cases
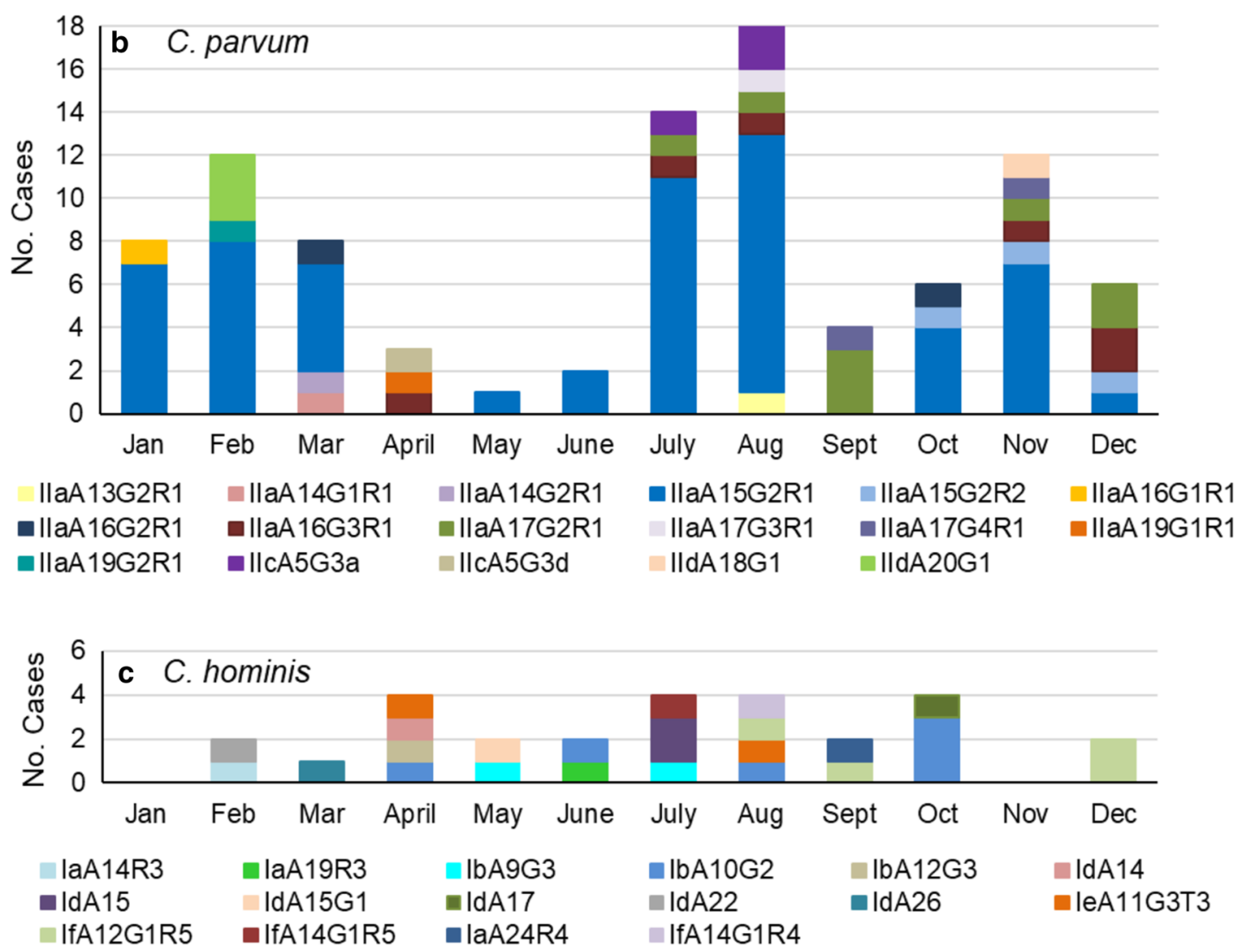

Fig. 2 Total cryptosporidiosis cases in Ontario and subtypes of the gp60 gene of a subset of Cryptosporidium parvum and C. hominis cases, diagnosed by month for the periods from 2008-2017. a Total monthly cases of cryptosporidiosis reported in Ontario versus subtyped cases of $C$. parvum and C. hominis from 2008-2017 (excluding 2010); b monthly occurrence of 94 gp60 subtypes from cases with single and mixed C. parvum; c monthly occurrence of $27 \mathrm{gp} 60$ subtypes from cases with single and mixed cases of C. hominis

observed a higher peak of cases in females from 20-35 years old, which could potentially coincide with reproductive years, during which time child rearing could theoretically enhance risk of exposure. This age distribution was not observed in PEI; instead, a second peak was noted among those aged $45-50$ years [17].
Differences in C. parvum and C. hominis geographical distribution exist in some regions, with higher $C$. hominis in urban centers and C. parvum in rural regions [32, 40]. Maier et al. [41] reported that people living in rural areas had twice the odds of having Cryptosporidium than people living in urban settings; however, molecular data were 
Table 3 Proportion of C. parvum llaA15G2R1 gp60 subtype identified per year in cryptosporidiosis cases from Ontario

\begin{tabular}{llcl}
\hline Year & Total number reported cases & $\begin{array}{l}\text { Total number of C. parvum and C. hominis cases } \\
\text { subtyped }\end{array}$ & $\begin{array}{l}\text { Proportion of cases with } \\
\text { the llaA15G2R1 subtype } \\
(\%)\end{array}$ \\
\hline 2008 & & 1 & $0 / 1(0)$ \\
2009 & 338 & 1 & $0 / 1(0)$ \\
2011 & 309 & 5 & $3 / 5(60)$ \\
2012 & 301 & 21 & $8 / 21(38)$ \\
2013 & 299 & 8 & $5 / 8(63)$ \\
2014 & 309 & 5 & $0 / 5(0)$ \\
2015 & 362 & 32 & $13 / 32(41)$ \\
2016 & 387 & 26 & $17 / 26(65)$ \\
2017 & 429 & 18 & $12 / 18(67)$ \\
\hline
\end{tabular}

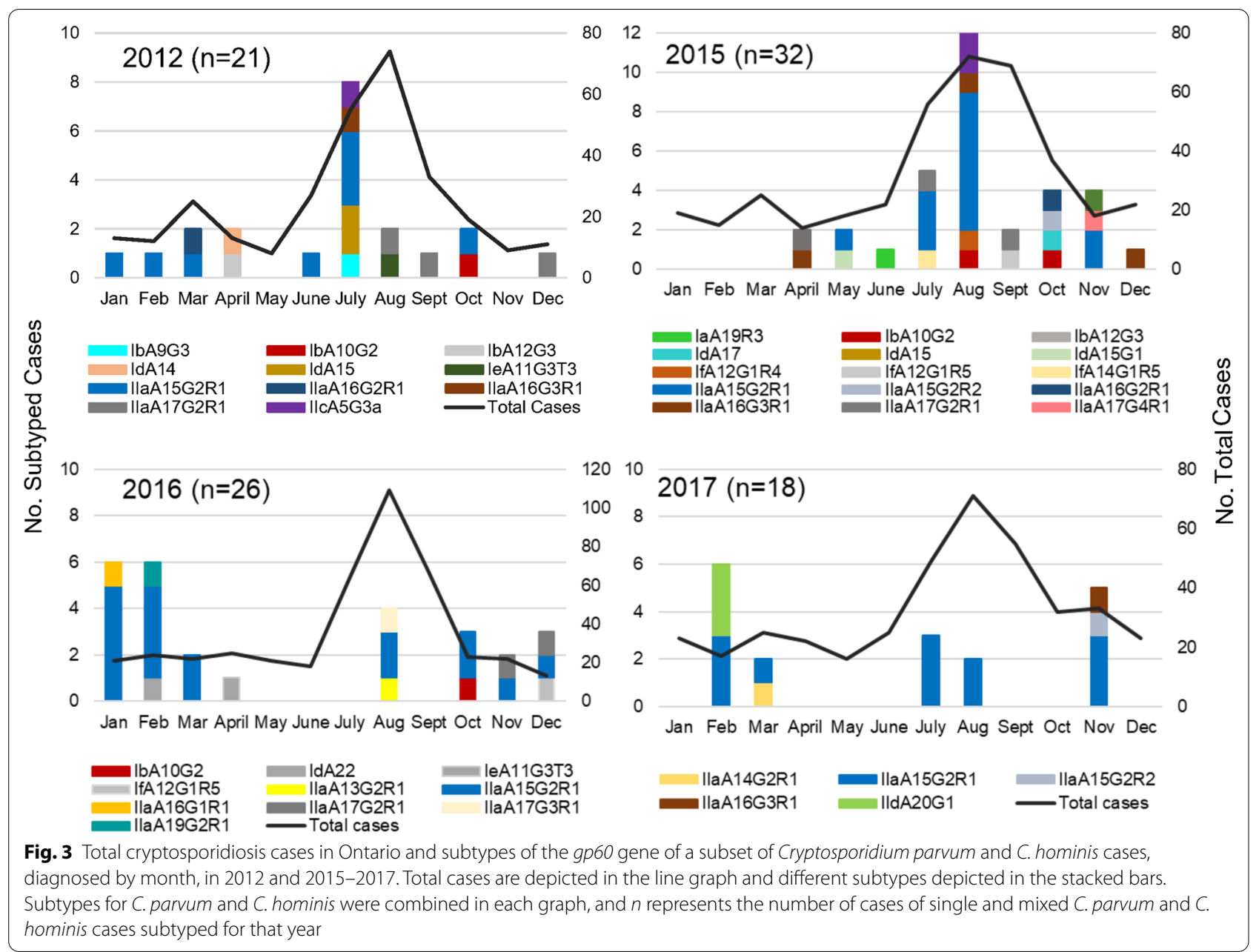

not available to determine the Cryptosporidium spp. in those cases. Information on place of residence for cases in our study was not provided in the dataset.

Cases of Cryptosporidium in Ontario peak in the summer months. By contrast, winter and late autumn peaks were evident among the sub-typed cases in addition to the more common summer peak. This bias in 'seasonal' distribution may be because some of the specimens provided for subtyping were obtained from a biobank where some unpreserved specimens, of the same case as 
Table 4 Summary of gp60 subtypes reported in Canadians from five Provinces and one Territory

\begin{tabular}{|c|c|c|c|c|c|c|c|}
\hline Subtypes & $\left.P E\right|^{h}$ & Quebec & Nunavut & Ontario & Saskatchewan & $B C^{i}$ & Total \\
\hline C. hominis & & & & & & & 71 \\
\hline laA14R3 & - & - & - & $1 / 27^{f}$ & - & - & 1 \\
\hline laA19R3 & - & - & - & $1 / 27^{f} ; 1 / 4^{e}$ & - & - & 2 \\
\hline laA22R3 & - & $1 / 11^{c}$ & - & - & - & - & 1 \\
\hline laA23R4 & - & - & - & $1 / 4^{e}$ & - & - & 1 \\
\hline laA24R4 & - & - & - & $1 / 27^{f}$ & - & - & 1 \\
\hline IaA25R3 & - & - & - & $1 / 27^{f}$ & - & - & 1 \\
\hline IbA9G3 & - & $1 / 11^{c}$ & - & $2 / 27^{f}$ & - & - & 3 \\
\hline IbA10G2 & - & $6 / 11^{c}$ & - & $6 / 27^{f}$ & $2 / 11^{g}$ & - & 14 \\
\hline $\mathrm{IbA12G3}$ & - & - & - & $1 / 27^{f}$ & - & - & 1 \\
\hline $\mathrm{IdA} 13$ & - & $1 / 14^{b}$ & - & - & - & - & 1 \\
\hline $\mid \mathrm{dA} 14$ & - & $5 / 14^{b}$ & - & $1 / 27^{f}$ & - & - & 6 \\
\hline $\mathrm{IdA14G1}$ & - & $1 / 14^{b}$ & - & - & - & - & 1 \\
\hline IdA14G2R1 & - & $1 / 14^{b}$ & - & - & - & - & 1 \\
\hline IdA15 & - & $5 / 14^{b}$ & - & $2 / 27^{f}$ & - & - & 7 \\
\hline IdA15G1 & - & - & - & $1 / 27^{f}$ & - & - & 1 \\
\hline $\mathrm{IdA} 16$ & - & $1 / 14^{b}$ & - & - & - & - & 1 \\
\hline IdA17 & - & - & - & $1 / 27^{f}$ & - & - & 1 \\
\hline IdA17G1 & - & $2 / 11^{c}$ & - & - & - & - & 2 \\
\hline $\mid d A 18$ & - & - & - & - & - & $4 / 4^{9}$ & 4 \\
\hline $\mathrm{IdA} 19$ & - & $1 / 11^{c}$ & - & $1 / 4^{e}$ & $9 / 11^{\mathrm{g}}$ & - & 11 \\
\hline IdA22 & - & - & - & $1 / 27^{f}$ & - & - & 1 \\
\hline IdA26 & - & - & - & $1 / 27^{f}$ & - & - & 1 \\
\hline leA11G3T3 & - & - & - & $2 / 27^{f} ; 1 / 4^{e}$ & - & - & 3 \\
\hline IfA12G1R4 & - & - & - & $1 / 27^{f}$ & - & - & 1 \\
\hline IfA12G1R5 & - & - & - & $3 / 27^{f}$ & - & - & 3 \\
\hline IfA14G1R5 & - & - & - & $1 / 27^{f}$ & - & - & 1 \\
\hline C. parvum & & & & & & & 133 \\
\hline IlaA13G2R1 & - & - & - & $1 / 94^{f}$ & - & - & 1 \\
\hline IlaA14G1R1 & - & - & - & $1 / 94^{f}$ & - & - & 1 \\
\hline IlaA14G2R1 & - & - & - & $1 / 94^{f}$ & - & - & 1 \\
\hline IlaA15R1 & - & - & $1 / 7^{d}$ & - & - & - & 1 \\
\hline IlaA15G1R1 & - & - & $2 / 7^{d}$ & - & - & - & 2 \\
\hline IlaA15G2R1 & $1 / 5^{\mathrm{a}}$ & $13 / 24^{c}$ & $3 / 7^{d}$ & $58 / 94^{f}$ & - & - & 75 \\
\hline IlaA15G2R2 & - & - & - & $3 / 94^{f} ; 1 / 4^{e}$ & - & - & 4 \\
\hline IlaA16G1R1 & - & - & $1 / 7^{d}$ & $1 / 94^{f}$ & - & - & 2 \\
\hline IlaA16G2R1 & $4 / 5^{\mathrm{a}}$ & $3 / 24^{c}$ & - & $2 / 94^{f}$ & - & - & 9 \\
\hline IlaA16G3R1 & - & $4 / 24^{c}$ & - & $6 / 94^{\mathrm{f}} ; 1 / 4^{\mathrm{e}}$ & - & - & 11 \\
\hline IlaA17G2R1 & - & $2 / 24^{c}$ & - & $8 / 94^{\mathrm{f}} ; 2 / 4^{\mathrm{e}}$ & - & - & 12 \\
\hline IlaA17G3R1 & - & $2 / 24^{c}$ & - & $1 / 94^{f}$ & - & - & 3 \\
\hline IlaA17G4R1 & - & - & - & $2 / 94^{f}$ & - & - & 2 \\
\hline IlaA19G1R1 & - & - & - & $1 / 94^{f}$ & - & - & 1 \\
\hline IlaA19G2R1 & - & - & - & $1 / 94^{f}$ & - & - & 1 \\
\hline IIcA5G3a & - & - & - & $3 / 94^{f}$ & - & - & 2 \\
\hline IIcA5G3d & - & - & - & $1 / 94^{f}$ & - & - & 1 \\
\hline IIdA18G1 & - & - & - & $1 / 94^{f}$ & - & - & 1 \\
\hline IIdA20G1 & - & - & - & $3 / 94^{f}$ & - & - & 3 \\
\hline
\end{tabular}

The following numbers represent the references cited: ${ }^{\mathrm{a}} 17 ;{ }^{\mathrm{b}} 6 ;{ }^{\mathrm{c}} 7 ;{ }^{\mathrm{d}} 5 ;{ }^{\mathrm{e}} 18 ;{ }^{\mathrm{f}}$ present study; ${ }^{\mathrm{g}} 31 .{ }^{\mathrm{h}} \mathrm{PEI}=$ Prince Edward Island; ${ }^{\mathrm{i}} \mathrm{BC}=\mathrm{British}$ Columbia; subtypes in bold are the first reported occurrence in Canada 
Cryptosporidium positive specimens, were submitted for testing of other enteric pathogens. Insulander et al. [11] reported that people who develop diarrhea after traveling abroad might be more likely to seek medical attention. Travel of many Canadians to the Caribbean and Central America during late fall and winter may in part account for the higher percentage of unpreserved specimens obtained in this study for subtyping during that period. Nonetheless, results of the present study showed a summer peak of cryptosporidiosis due to C. parvum, which aligns with summer peaks of cryptosporidiosis cases from passive surveillance data in Ontario $[19,20]$ and surveillance data from Quebec [7]. This is in contrast to PEI where peak human infections with $C$. parvum were in April-June, corresponding with the time of year of cattle shedding, whereas a late summer/early autumn peak mainly involved C. hominis cases [17]. This trend also occurs in England, Wales and Scotland where spring peaks are associated with $C$. parvum and September peaks are associated with $C$. hominis $[12,32,35,42]$.

A study of cryptosporidiosis in cattle (2008-2014) and humans (2009-2015) in southwestern Ontario [43] revealed a peak shedding of Cryptosporidium in cattle in late winter/early spring and a peak in human cases of Cryptosporidium in mid-summer. Considering environmental conditions, a delay between shedding and environmental exposure through recreational water use was estimated to be a reasonable mechanism of zoonotic transfer of Cryptosporidium in the region of Ontario [43]. This may help to explain the summer peak of C. parvum cases observed in the present study.

The gp60 subtype IIaA15G2R1 is the most frequently observed C. parvum subtype in humans and calves globally [9] and was the most frequently observed subtype in the current study and in neighboring Quebec [7]. In the USA, the highest rates of cryptosporidiosis are seen in the upper Midwest of the country where predominant subtypes are IIaA15G2R1 in Wisconsin [15] and IIaA16G2R1 in Michigan [14]. In Northern Europe, predominant subtypes are IIaA15G2R1 in Scotland [12], IIaA17G2R1 and IIaA15G2R1 in England and Wales $[35,44]$ and IIaA18G3R1 in Ireland [10]. In Sweden, IIaA15G2R1 was mainly associated with travel outside the country [11]. The first molecular epidemiology study of cryptosporidiosis in humans and in neonatal dairy calves in Ontario revealed similar gp60 subtypes in $C$. parvum isolates: 7 subtypes in 44 isolates [18]. Only 11 unpreserved human isolates were available from 20032004, and 8 were successfully typed, revealing four $C$. parvum IIa isolates (one IIaA15G2R2, two IIaA17G2R1 and one IIaA16G3R1); all three subtypes were identified in the current study. Interestingly, IIaA15G2R1, one of three predominant subtypes in the Ontario calves, and observed on 10/16 farms in South Western Ontario [18], was not observed among their four human isolates. We observed IIaA15G2R2 in three cases. In PEI, subtype IIaA16G2R1 represented 55\% of the cattle isolates, and subtypes IIaA16G3R1 and IIaA15G2R1 represented 22\% of the isolates each [17].

A high rate of cryptosporidiosis in Nunavik, Northern Canada, was observed with $15.7 \%$ prevalence at a hospital where 108 diarrheal stool samples were analyzed [5]. Seven samples were typed, and all belonged to the C. parvum IIa subfamily; three of the seven were the IIaA15G2R1. It is interesting to speculate on the source of this subtype as livestock are not reared in the far north of Canada. Seals may be a potential source of infection as C. parvum has been found in seals in Nunavut [45].

The zoonotic IIaA17G2R1 subtype, the second most frequent C. parvum subtype in this study, is commonly found in calves and is strongly linked with visits to farms and farm animal contact [44]. It is a predominant subtype in England and Wales [44] and was reported in Ontario and Quebec [7, 18]. IIaA17G2R1 has been found in rodents in Thailand, which suggests rats may be a source of infection [46]. Another zoonotic genotype observed in this study was the IId isolate, a subtype that is hostadapted to small ruminants such as lambs and goats and found in humans $[9,44]$.

Our data-particularly the finding that C. parvum dominated in the samples-support that animal contact may be another important route of transmission in Ontario. Indeed, a Canadian study estimated the annual burden of enteric illness associated with animal contact revealed that Cryptosporidium had the highest proportion of illnesses attributable to animal contact of the eight enteric pathogens studied [4].

While potentially zoonotic subtypes predominated in this study, human-host adapted C. hominis was observed in $18.6 \%$ of cases, and the human host-adapted IIc subfamily of C. parvum [9] in three cases, suggesting anthroponotic transmission likely occurred in about a quarter of cases analyzed. IbA10G2, a subtype associated with drinking and recreational water outbreaks in Canada [31], was the most frequently reported $C$. hominis subtype in this study. Thus, recreational water and drinking water are likely sources of this subtype and are known risk factors in Ontario [19]. Trotz-Williams [18], in their study in Ontario, Canada, did not observe the IbA10G2 subtype; instead, they reported four $C$. hominis subtypes (IaA19R3, IaA23R4, IdA19 and IeA11G3T3), two of which (IaA19R3 and IeA11G3T3) were observed in this study. The IdA19 subtype they observed has been associated with swimming pool and drinking water outbreaks elsewhere in Canada [31]. 
IbA10G2 is the most frequent subtype of $C$. hominis reported in European countries [11, 34, 44]. This subtype was predominant in the USA, until a new subtype, IaA28R4, emerged as the major water-associated subtype in the USA in 2005 [25]. Since 2013, subtype IfA12G1R5 has emerged as the predominant $C$. hominis subtype in sporadic and outbreak cases in the USA [47] and has emerged as one of two dominant subtypes in Western Australia [39]. IfA12G1R5 was observed in three cases in the present study, whereas IaA28R4 has not been reported in Canada to date.

Travel is frequently associated with cases of $C$. hominis in Northern Europe, especially subtypes other than the IbA10G2, in Sweden, England and Wales [11, 48]. Forty percent of unusual cases in England and Wales were associated with travel [49]. While travel was indicated for $4 / 129$ cases in this study, we cannot rule out that other cases were not travel associated, especially in light of previous reports that a quarter of the cryptosporidiosis cases in Ontario are associated with travel $[19,20]$. Thus, C. hominis infections in Ontario may be acquired during travel or through use of recreational or drinking water.

Two C. parvum cases were acquired during travel in this study. One case, acquired during travel to Romania, was IIaA19G1R1, a rare subtype associated with calves, lambs and goats and has been linked to several outbreaks in petting farms with lambs and goats in Norway [52] and a lamb in England [53]. The other case was with IIaA17G2R1.

Other C. hominis subtypes observed in the present study were one case of IdA14, two cases of IdA15 and one of IdA15G1. The Id subfamily was the most frequently observed subfamily in an outbreak of cryptosporidiosis in Nunavik, northern Canada [6]. The authors reported five Id subtypes including IdA14 and IdA15 that were observed in this study. A longitudinal study in Western Australia revealed the predominant $C$. hominis subtype was IdA15G1, occurring in $45 \%$ of cases [33]. This subtype has been associated with Australian outbreaks in 2007 [50], has a higher prevalence in Aboriginal populations [51] and is the predominant subtype in rural areas of Western Australia [39].

The largest analysis of outbreak data was recently published for England and Wales and demonstrates the power of molecular subtyping for understanding reservoirs of contamination and routes of transmission [54]. The authors demonstrated that IbA10G2 and IIaA15G2R1 predominated for C. hominis and C. par$v u m$, respectively, in outbreak isolates from England and Wales [54]. These are the major subtypes occurring in Canada; however, we did not have data on outbreak status for the 131 cases in this study. Subtyping of isolates from outbreaks in Canada is available for only a handful of studies, including the large drinking water outbreak in North Battleford, Saskatchewan, in 2001, which affected $>5000$ individuals. Both the IdA19 and IbA10G2 subtypes of $C$. hominis were identified in the outbreak cases [31]. The IdA19 has also been identified in a recreational water outbreak in BC [31]. Five Id subtypes were associated with an outbreak in villages in Nunavik in Northern Quebec; the source of the outbreak was not identified [6]. Cryptosporidium parvum is implicated in foodborne outbreaks such as an outbreak with subtype IIaA17G1R1 where chopped parsley was the suspected source [11] and subtypes IIaA15G2R1 and IIaA16G1R1 associated with a foodborne outbreak where arugula was the suspected source [11]. IIaA15G2R1 subtype is the dominant subtype of outbreaks in the USA [30].

While C. parvum and C. hominis comprised $92.2 \%$ of the cases in this study, several other species were identified at low frequencies. Cryptosporidium ubiquitum was the third most commonly identified species with five cases. Ong et al. [55] were the first to describe C. ubiquitum (previously referred to as the cervine genotype) and its association with a swimming pool outbreak in British Columbia (Canada). Trotz-Williams [18] reported a single human case associated with C. ubiquitum that was genotyped as the XIIa subfamily, commonly associated with ruminants and described in humans in the UK [28]. The isolates in the current study were both the rodent types XIIb and XIId, subtypes reported in humans and environmental water, including drinking source water in Northeastern USA [28]. Recreational swimming in lakes and rivers is a known risk factor for cryptosporidiosis [19] and may be the route of infection with C. ubiquitum.

One of the two C. felis cases in this study was typable using the recently developed C. felis gp60 primers [26] and was subtype XIXd-1. The sequence was homologous with the Swec 053 isolate (GenBank: MH240831.1) from a Swedish traveler to India and clusters closely with 17 other human isolates of $C$. felis, mainly from the UK and Swedish travel cases. This cluster of cases is very conserved and distinct from other C. felis gp60 clusters [26]. To date, $17 / 18$ reported cases were in males (median age, 37 years) [26]; the case in this study was a male in his 30s.

Cryptosporidium meleagridis, a bird-associated species, is also responsible for about $10 \%$ of cryptosporidiosis cases globally, likely through zoonotic and anthroponotic transmission [56]. We observed one $C$. meleagridis case with the IIIeA21G2R1 subtype in a female who likely acquired the infection during travel to an undisclosed location. The IIIe subtype has been reported in Swedish travelers to Asia [11], rodents and chickens in Asia $[57,58]$ and red partridges in Slovakia [59]. Two GenBank submissions of IIIeA21G2R1 (accessed in September 2020) were from a Swedish 
traveler to Indonesia and a chicken in China (GenBank: KU852728.1 and KJ672091.1).

Cryptosporidium meleagridis, C. canis, C. felis and C. muris are more common in developing countries [9] and frequently reported in individuals with HIV and other immunodeficiencies $[36,58]$ and in immunocompromised individuals $[36,49,60]$. Data regarding immune status were unavailable for the $C$. meleagridis case, two C. felis cases and the C. muris case detected in this study.

A limitation of this study was the small sample sizes per year and the random selection of isolates characterized. Additionally, not every stool specimen submitted to Public Health Ontario has a corresponding unpreserved specimen, and we cannot exclude the possibility of differential submission patterns over time. Significant underreporting of symptoms and presentation bias may also influence the types of patients for whom a stool sample is even ordered. Important clinical data-including intercurrent immunosuppression, due to HIV, for example, as well as chronicity of symptoms-are almost universally missing from standard laboratory test requisitions. Thus, the cases represented in this analysis may lack external validity to all patients with cryptosporidiosis in the province of Ontario, and our comparisons may be skewed accordingly.

\section{Conclusions}

This study presents the largest number of cases typed to date in Canada and demonstrates a wide variety of Cryptosporidium subtypes present in Ontario cases. As only a fraction of yearly specimens (3-17\%) was tested, this study provides an estimate of the potential sources and transmission routes. This study supports previous epidemiological analyses in Ontario in showing that bovine isolates are potentially a large cause of cryptosporidiosis in Ontario [19, 20]. Differences may also occur through changes in patterns of infection over time such as the emergence of the IaA28R4 subtype as the major isolate associated with USA recreational water outbreaks when IbA10G2 predominated prior to 2006. National surveillance programs such as in the UK or the USA CryptoNet program enable detection of changes over time that may provide mitigation and prevention strategies $[25,32]$. Routine national molecular surveillance of cryptosporidiosis is more feasible now as there has been a switch to molecular testing in many primary laboratories in Ontario [61] and in other parts of Canada; thus, unpreserved specimens would be more readily available for molecular analyses.

While gp60 is the most widely used marker globally for subtyping Cryptosporidium, further subtyping is required to discriminate gp60 subtypes, such as the common IIaA15G2R1. Numerous other markers have been identified; however, additional markers will provide greater discrimination of sources of IIaA15G2R1 and other subtypes in humans. Research on wholegenome sequence (WGS) comparisons of diverse human and animal isolates is ongoing, and more WGS data are needed to support identification of new markers to provide robust discrimination between sources, identify risk factors and elucidate patterns of transmission [62]. Ultimately, a harmonized multi-locus typing scheme would provide better analysis of transmission not only in Europe as proposed [63] but also globally.

\section{Abbreviations}

SSU rRNA: Small subunit ribosomal ribonucleic acid; gp60: Glycoprotein 60; nPCR: Nested polymerase chain reaction; PEl: Prince Edward Island; BC: British Columbia; WGS: Whole genome sequencing.

\section{Acknowledgements}

The authors thank Stephanie Murphy from the Parasite Biology Unit of the National Microbiology Laboratory at Guelph for her technical support in this study and Laura Martin, from the same unit, for critical review of the manuscript. The authors thank the Clinical Parasitology Department at the Public Health Ontario Laboratory.

\section{Authors' contributions}

RAG conceived the study and was primarily responsible for data analysis, molecular analyses and data interpretation, and for drafting the manuscript. CAY performed molecular testing and data analysis, developed and applied the bioinformatics CryptoGenotyper program and contributed to critical appraisal of the manuscript; PKM contributed to epidemiological and statistical analysis of the study, interpretation and critical appraisal of the manuscript; KT contributed to interpretation and critical appraisal of the manuscript. MAR performed molecular testing and data analysis and to critical appraisal of the manuscript. RL contributed to data collection and analysis and to critical appraisal of the manuscript. AKB contributed to data collection, analysis and interpretation, and to critical revision of the manuscript. All authors read and approved the final manuscript.

Funding

This work was funded by the Public Health Agency of Canada.

\section{Availability of data and materials}

The datasets generated during and/or analysed during the current study are not publicly available due to privacy restrictions and ethical considerations but are available from the corresponding author on reasonable request.

\section{Ethics approval and consent to participate}

Ethics approval was granted by the Research Ethics Board of Health Canada/ Public Health Agency of Canada (Ethics Certificate REB 2016-010P). Specimens were transferred to the Public Health Agency of Canada from Public Health Ontario in accordance with a Material Transfer Agreement (MTA 16-033).

\section{Consent for publication}

All authors read and approved the final manuscript

\section{Competing interests}

The authors have no competing interests.

\section{Author details}

1 Parasite Biology Unit/Division of Enteric Diseases, National Microbiology Laboratory, Public Health Agency of Canada, 110 Stone Road West, Guelph, ON N1G 3W4, Canada. ${ }^{2}$ Centre for Food-borne, Environmental \& Zoonotic Infectious Diseases, Public Health Agency of Canada, 370 Woodlawn Road West, Guelph, ON N1H 7M7, Canada. ${ }^{3}$ Laboratoire de santé publique du Québec, Institut national de santé publique du Québec, 20045, chemin Sainte-Marie, Sainte-Anne-de-Bellevue, Québec H9X 3R5, Canada. ${ }^{4}$ Public 
Health Ontario Laboratory, Public Health Ontario, Toronto M5G 1M1, Canada.

${ }^{5}$ Tropical Disease Unit, Toronto General Hospital, Toronto M5G 2C4, Canada.

${ }^{6}$ Faculty of Medicine, University of Toronto, Toronto M5S 1A8, Canada.

Received: 4 September 2020 Accepted: 13 December 2020

Published online: 22 January 2021

\section{References}

1. Kotloff KL, Nasrin D, Blackwelder WC, Wu Y, Farag T, Panchalingham S, et al. The incidence, aetiology, and adverse clinical consequences of less severe diarrhoeal episodes among infants and children residing in lowincome and middle-income countries: a 12-month case-control study as a follow-on to the Global Enteric Multicenter Study (GEMS). Lancet Glob HIth. 2019;7:e568-84.

2. Khalil IA, Troeger C, Rao PC, Blacker BF, Brown A, Brewer TG, et al. Morbidity, mortality, and long-term consequences associated with diarrhoea from Cryptosporidium infection in children younger than 5 years: a metaanalyses study. Lancet Glob HIth. 2018;6:e758-68.

3. Canadian Notifiable Disease Surveillance System (CNDSS). Case definitions: Nationally notifiable diseases (https://diseases.canada.ca/notifiable /diseases-list).

4. Murray R, Tataryn J, Pintar K, Thomas MK. Estimates of the burden of illness for eight enteric pathogens associated with animal contact in Canada. Epidemiol Infect. 2017;145:3413-23.

5. Iqbal A, Goldfarb DM, Slinger R, Dixon BR. Prevalence and molecular characterization of Cryptosporidium spp. and Giardia duodenalis in diarrhoeic patients in the Qikiqtani Region, Nunavut, Canada. Int J Circumpol HIth. 2015;19:27713.

6. Thivierge K, labal A, Dixon B, Dion R, Levesque B, Cantin P, et al. Cryptosporidium hominis is a newly recognized pathogen in the arctic region of Nunavik, Canada: molecular characterization of an outbreak. PLoS Negl Trop Dis. 2016;10:e0004534.

7. Hutter JA, Dion R, Irace-Cima A, Fiset M, Guy RA, Dixon B, et al. Cryptosporidium spp.: human incidence, molecular characterization and associated exposures in Québec, Canada (2016-2017). PLoS ONE. 2020; 15:e0228986

8. Cacciò SM, Chalmers RM. Human cryptosporidiosis in Europe. Clin Microbiol Infect. 2016;22:471-80.

9. Feng Y, Ryan UM, Xiao L. Genetic diversity and population structure of Cryptosporidium. Trends Parasitol. 2018;34:997-1011.

10. Zintl A, Ezzaty-Mirashemi M, Chalmers RM, Elwin K, Mulcahy G, Lucy FE, et al. Longitudinal and spatial distribution of GP60 subtypes in human cryptosporidiosis cases in Ireland. Epidemiol Infect. 2011;139:1945-55.

11. Insulander M, Silverlås C, Lebbad M, Karlsson L, Mattsson JG, Svenungsson B. Molecular epidemiology and clinical manifestations of human cryptosporidiosis in Sweden. Epidemiol Infect. 2013;141:1009-20.

12. Deshpande AP, Jones BL, Connelly L, Pollock KG, Brownlie S, Alexander CL. Molecular characterization of Cryptosporidium parvum isolates from human cryptosporidiosis cases in Scotland. Parasitol. 2015;142:318-25.

13. Stensvold CR, Ethelberg S, Hansen L, Sahar S, Voldstedlund M, Kemp M, et al. Cryptosporidium infections in Denmark, 2010-2014. Dan Med J. 2015;2:A5086.

14. Peng MM, Wilson ML, Holland RE, Meshnick SR, Lal AA, Xiao L. Genetic Diversity of Cryptosporidium Spp. in cattle in Michigan: implications for understanding the transmission dynamics. Parasitol Res. 2003;90:175-80.

15. Feltus DC, Giddings CW, Schneck BL, Monson T, Warshauer D, McEvoy JM. Evidence supporting zoonotic transmission of Cryptosporidium spp. Wisconsin. J Clin Microbiol. 2006:44:4303-8.

16. Xiao L, Zhou L, Santin M, Yang W, Fayer R. Distribution of Cryptosporidium parvum subtypes in calves in eastern United States. Parasitol Res. 2007;100:701-6.

17. Budu-Amoako E, Greenwood SJ, Dixon BR, Sweet L, Ang L, Barkema HW, et al. Molecular epidemiology of Cryptosporidium and Giardia in humans on Prince Edward Island, Canada: evidence of zoonotic transmission from cattle. Zoon Pub Hlth. 2012:59:424-33.

18. Trotz-Williams LA, Martin DS, Gatei W, Cama V, Peregrine AS, Martin SW, et al. Genotype and subtype analyses of Cryptosporidium isolates from dairy calves and humans in Ontario. Parasitol Res. 2006;99:346-52.
19. Pintar KD, Pollari F Waltner-Toews D, Charron DF, McEwen SA, Fazil A, et al. A modified case-control study of cryptosporidiosis (using nonCryptosporidium-infected enteric cases as controls) in a community setting. Epidemiol Infect. 2009;137:1789-99.

20. Vrbova L, Johnson K, Whitfield Y, Middleton D. A descriptive study of reportable gastrointestinal illnesses in Ontario, Canada, from 2007 to 2009. BMC Pub HIth. 2012:12:970.

21. Public Health Ontario. Infectious Disease Trends (https://www.publi chealthontario.ca/en/data-and-analysis/infectious-disease/reportable -disease-trends-annually).

22. Xiao L, Alderisio K, Limor J, Royer M, Lal AA. Identification of species and sources of Cryptosporidium oocysts in storm waters with a smallsubunit rRNA-based diagnostic and genotyping tool. Appl Environ Microbiol. 2000;66:5492-8.

23. Sulaiman IM, Morgan UM, Thompson RC, Lal AA, Xiao L. Phylogenetic relationships of Cryptosporidium parasites based on the 70-kilodalton heat shock protein (HSP70) gene. Appl Environ Microbiol. 2000;66:2385-91.

24. Moore CE, Elwin K, Phot N, Seng C, Mao S, Suy K, et al. Molecular characterization of Cryptosporidium species and Giardia duodenalis from symptomatic Cambodian children. PLoS Negl Trop Dis. 2016;10:e0004822

25. Xiao L, Hlavsa MC, Yoder J, Ewers C, Dearen T, Yang W, et al. Subtype analysis of Cryptosporidium specimens from sporadic cases in Colorado, Idaho, New Mexico, and lowa in 2007: widespread occurrence of one Cryptosporidium hominis subtype and case history of an infection with the Cryptosporidium horse genotype. J Clin Microbiol. 2009;47:3017-20.

26. Rojas-Lopez L, Elwin K, Chalmers RM, Enemark HL, Beser J, Troell K. Development of a gp60-subtyping Method for Cryptosporidium felis. Parasit Vectors. 2020;13:39.

27. Stensvold CR, Beser J, Axén C, Lebbad M. High applicability of a novel method for gp60-based subtyping of Cryptosporidium meleagridis. J Clin Microbiol. 2014:52:2311-9.

28. Li N, Xiao L, Alderisio K, Elwin K, Cebelinski E, Chalmers R, et al. Subtyping Cryptosporidium ubiquitum, a zoonotic pathogen emerging in humans. Emerg Infect Dis. 2014;20:217-24.

29. Yanta CA, Bessonov K, Robinson G, Troell K, Guy RA. CryptoGenotyper: a new bioinformatics tool for rapid Cryptosporidium identification. Submitted to Food Waterborne Parasitol.

30. Xiao L. Molecular epidemiology of cryptosporidiosis: an update. Exp Parasitol. 2010;124:80-9.

31. Ong CS, Chow S, Gustafson R, Plohman C, Parker R, Isaac-Renton JL, et al. Rare Cryptosporidium hominis subtype associated with aquatic center use. Emerg Infect Dis. 2008;14:1323-5.

32. Chalmers RM, Elwin $\mathrm{K}$, Thomas AL, Guy EC, Mason B. Long-term Cryptosporidium typing reveals the aetiology and species-specific epidemiology of human cryptosporidiosis in England and Wales, 2000 to 2003. Euro Surveill. 2009;14:19086

33. Ng J, MacKenzie B, Ryan U. Longitudinal multi-locus molecular characterisation of sporadic Australian human clinical cases of cryptosporidiosis from 2005 to 2008. Exp Parasitol. 2010;125:348-56.

34. de Lucio A, Merino FJ, Martínez-Ruiz R, Bailo B, Aguilera M, Fuentes I, et al. Molecular genotyping and sub-genotyping of Cryptosporidium spp. isolates from symptomatic individuals attending two major public hospitals in Madrid, Spain. Infect Genet Evol. 2016:37:49-56.

35. Chalmers RM, Smith R, Elwin K, Clifton-Hadley FA, Giles M. Epidemiology of anthroponotic and zoonotic human cryptosporidiosis in England and Wales, 2004-2006. Epidemiol Infect. 2011;139:700-12.

36. Laboratory-based surveillance for Cryptosporidium in France. 2006-2009 ANOFEL Cryptosporidium National Network. Euro Surveill. 2010:15:19642.

37. Wielinga PR, de Vries A, van der Goot TH, MankT, Mars MH, Kortbeek LM, et al. Molecular epidemiology of Cryptosporidium in humans and cattle in The Netherlands. Int J Parasitol. 2008;38:809-17.

38. Nic Lochlainn LM, Sane J, Schimmer B, Mooij S, Roelfsema J, van Pelt W, et al. Risk factors for sporadic cryptosporidiosis in the Netherlands: Analysis of a 3-year population based case-control study coupled with genotyping, 2013-2016. J Infect Dis. 2019;219:1121-9.

39. Braima K, Zahedi A, Oskam C, Reid S, Pingault N, Xiao L, et al. Retrospective analysis of Cryptosporidium species in Western Australian human populations (2015-2018), and emergence of the C. hominis IfA12G1R5 subtype. Infect Genet Evol. 2019:73:306-13. 
40. Loeck BK, Pedati C, Iwen PC, McCutchen E, Roellig DM, Hlavsa MC, et al. Genotyping and subtyping Cryptosporidium to identify risk factors and transmission patterns-Nebraska, 2015-2017. Morb Mortal Wkly Rep. 2020;69:335-8.

41. Maier A, Krolik J, Majury A. Triage and protocol recommendations for the parasitology laboratory based on an epidemiological investigation of parasite diagnostics in Ontario laboratories. Can J Infect Dis Med Microbiol. 2014;25:305-10.

42. Deshpande A, Alexander CL, Coyne M, Brownlie S, Smith-Palmer A, Jones BL. Molecular diversity of Scottish Cryptosporidium hominis isolates. Epidemiol Infect. 2015;143:1219-24.

43. Brankston G, Boughen C, Ng V, Fisman DN, Sargeant JM, Greer AL. Assessing the impact of environmental exposures and Cryptosporidium infection in cattle on human incidence of cryptosporidiosis in Southwestern Ontario, Canada. PLoS One. 2018;13:e0196573.

44. Chalmers RM, Smith RP, Hadfield SJ, Elwin K, Giles M. Zoonotic linkage and variation in Cryptosporidium parvum from patients in the United Kingdom. Parasitol Res. 2011;108:1321-5.

45. Appelbee AJ, Thompson RCA, Measures LM, Olson ME. Giardia and Cryptosporidium in harp and hooded seals from the Gulf of St. Lawrence, Canada. Vet Parasitol. 2010;173:19-23.

46. Tan TK, Low VL, Ng WH, Ibrahim J, Wang D, Tan CH. Occurrence of zoonotic Cryptosporidium and Giardia duodenalis species/genotypes in urban rodents. Parasitol Int. 2019;69:110-3.

47. Hlavsa MC, Roellig DM, Seabolt MH, Kahler AM, Murphy JL, McKitt TK, et al. Using molecular characterization to support investigations of aquatic facility-associated outbreaks of cryptosporidiosis - Alabama, Arizona, and Ohio, 2016. Morb Mortal Wkly Rep. 2017;66:493-7.

48. Chalmers RM, Hadfield SJ, Jackson CJ, Elwin K, Xiao L, Hunter P. Geographic linkage and variation in Cryptosporidium hominis. Emerg Infect Dis. 2008:14:496-8.

49. Elwin K, Hadfield SJ, Robinson G, Chalmers RM. The epidemiology of sporadic human infections with unusual cryptosporidia detected during routine typing in England and Wales, 2000-2008. Epidemiol Infect. 2012:140:673-83.

50. Ng-Hublin JSY, Combs B, Reid S, Ryan U. Comparison of three cryptosporidiosis outbreaks in Western Australia: 2003, 2007 and 2011. Epidemiol Infect. 2018;146:1413-24

51. Ng-Hublin JSY, Combs B, Reid S, Ryan U. Differences in the occurrence and epidemiology of cryptosporidiosis in aboriginal and non-aboriginal people in Western Australia (2002-2012). Infect Genet Evol. 2017:53:100-6.

52. Lange $H$, Johansen $\mathrm{OH}$, Vold L, Robertson LJ, Anthonisen IL, Nygard K. Second outbreak of infection with a rare Cryptosporidium parvum genotype in schoolchildren associated with contact with lambs/goat kids at a holiday farm in Norway. Epidemiol Infect. 2014;142:2105-13.
53. Utsi L, Smith SJ, Chalmers RM, Padfield S. Cryptosporidiosis outbreak in visitors of a UK industry-compliant petting farm caused by a rare Cryptosporidium parvum subtype: a case-control study. Epidemiol Infect. 2016;144:1000-9.

54. Chalmers RM, Robinson G, Elwin K, Elson R. Analysis of the Cryptosporidium spp. and gp60 subtypes linked to human outbreaks of cryptosporidiosis in England and Wales. to 2017. Parasit Vect. 2009;2019(12):95.

55. Ong CS, Eisler DL, Alikhani A, Fung VW, Tomblin J, Bowie WR, et al. Novel Cryptosporidium genotypes in sporadic cryptosporidiosis cases: first report of human infections with a cervine genotype. Emerg Infect Dis. 2002;8:263-8

56. Wang Y, Yang W, Cama V, Wang L, Cabrera L, Ortega Y, et al. Population genetics of Cryptosporidium meleagridis in humans and birds: evidence for cross-species transmission. Int J Parasitol. 2014;44:515-21.

57. Liao C, Wang T, Koehler AV, Fan Y, Hu M, Gasser RB. Molecular investigation of Cryptosporidium in farmed chickens in Hubei Province, China, identifies 'zoonotic' subtypes of C. meleagridis. Parasit Vect. 2018;11:484.

58. Sannella AR, Suputtamongkol Y, Wongsawat E, Cacciò SM. A retrospective molecular study of Cryptosporidium species and genotypes in HIVinfected patients from Thailand. Parasit Vect. 2019;12:91.

59. Máca O, Pavlásek I. First finding of spontaneous infections with Cryptosporidium baileyi and C. meleagridis in the red-legged partridge Alectoris rufa from an aviary in the Czech Republic. Vet Parasitol. 2015;209:164-8.

60. Wang RJ, Li JQ, Chen YC, Zhang LX, Xiao LH. Widespread occurrence of Cryptosporidium infections in patients with HIV/AIDS: Epidemiology, clinical feature, diagnosis, and therapy. Acta Trop. 2018;187:257-63.

61. Johnson KO, Thayani P, Warshawsky B, Whitfield Y, Pritchard J, Murti M. The epidemiology of cryptosporidiosis in Ontario, Canada following the introduction of PCR testing in 2018. Can Commun Dis Rep. 2020;46:227-30.

62. Fan Y, Feng Y, Xiao L. Comparative genomics: how has it advanced our knowledge of cryptosporidiosis epidemiology? Parasitol Res. 2019;118:3195-204.

63. Chalmers RM, Pérez-Cordón G, Cacció SM, Klotz C, Robertson LJ; participants of the Cryptosporidium genotyping workshop (EURO-FBP). Cryptosporidium genotyping in Europe: The current status and processes for a harmonised multi-locus genotyping scheme. Exp Parasitol. 2018;191:25-30.

\section{Publisher's Note}

Springer Nature remains neutral with regard to jurisdictional claims in published maps and institutional affiliations.

Ready to submit your research? Choose BMC and benefit from:

- fast, convenient online submission

- thorough peer review by experienced researchers in your field

- rapid publication on acceptance

- support for research data, including large and complex data types

- gold Open Access which fosters wider collaboration and increased citations

- maximum visibility for your research: over $100 \mathrm{M}$ website views per year

At BMC, research is always in progress.

Learn more biomedcentral.com/submissions 